MATHEMATICS OF COMPUTATION

Volume 73, Number 248, Pages 1779-1799

S $0025-5718(04) 01631-\mathrm{X}$

Article electronically published on January 23, 2004

\title{
NUMERICAL SCHEMES FOR THE SIMULATION OF THE TWO-DIMENSIONAL SCHRÖDINGER EQUATION USING NON-REFLECTING BOUNDARY CONDITIONS
}

\author{
XAVIER ANTOINE, CHRISTOPHE BESSE, AND VINCENT MOUYSSET
}

\begin{abstract}
This paper adresses the construction and study of a Crank-Nicolson-type discretization of the two-dimensional linear Schrödinger equation in a bounded domain $\Omega$ with artificial boundary conditions set on the arbitrarily shaped boundary of $\Omega$. These conditions present the features of being differential in space and nonlocal in time since their definition involves some time fractional operators. After having proved the well-posedness of the continuous truncated initial boundary value problem, a semi-discrete Crank-Nicolson-type scheme for the bounded problem is introduced and its stability is provided. Next, the full discretization is realized by way of a standard finite-element method to preserve the stability of the scheme. Some numerical simulations are given to illustrate the effectiveness and flexibility of the method.
\end{abstract}

\section{INTRODUCTION}

This paper is devoted to the numerical computation of the solution $u$ to the two-dimensional linear Schrödinger equation with constant coefficients

$$
\left\{\begin{array}{l}
\left(i \partial_{t}+\Delta\right) u(x, t)=0 \quad \forall(x, t) \in \mathbb{R}^{2} \times \mathbb{R}^{*+} \\
u(x, 0)=u_{0}(x) \quad \forall x \in \mathbb{R}^{2}
\end{array}\right.
$$

where $u_{0}$ designates the initial datum, $\Delta$ is the Laplace operator defined by $\Delta=$ $\partial_{x_{1}}^{2}+\partial_{x_{2}}^{2}$, and $x=\left(x_{1}, x_{2}\right)$ stands for the space variable. This kind of equation has many technological applications, e.g., in quantum mechanics (modeling of quantum devices [5]), in electromagnetic wave propagation [21], in underwater acoustics (paraxial approximations of the wave equation [28) or in optics (Fresnel equation [19]). When a non-linear perturbation is added, it can be used to model some problems arising in plasmas or relativistic physics but also for beam propagation in non-linear Kerr media [10. For all these reasons, the construction of efficient numerical schemes for solving (1.1) represents an important stake.

A standard discretization for system (1.1) is given by the well-known implicit Crank-Nicolson scheme. To bound the computational domain, one usually imposes a boundary condition of the Dirichlet or Neumann type. However, when the wave $u$ impinges the fictive boundary on which this boundary condition is set, some visible spurious reflections occur 12 and are prejudicial to the numerical observation of the propagation phenomenon. Then, one must consider a larger computational

Received by the editor November 7, 2002 and, in revised form, April 7, 2003.

2000 Mathematics Subject Classification. Primary 65M12, 35Q40, 58J40, 26A33, 58J47.

Key words and phrases. Schrödinger equation, non-reflecting boundary condition, stability, semi-discrete Crank-Nicolson-type scheme, finite-element methods.

(C)2004 American Mathematical Society 
domain which can be difficult to handle numerically, especially for multidimensional calculations. Therefore, a usual adopted solution consists in imposing a more suitable boundary condition on the fictive boundary which does not affect the solution in the interior domain by not generating any undesirable parasistic reflected waves. Numerous works have been devoted to this problem in the one-dimensional case. The ideal boundary condition, also often called exact, may be simply designed by the Laplace or Fourier transform [4, [6], [8], [9], 11], [14]. The resulting condition is then non-local in time and involves a temporal fractional derivative operator of order $1 / 2$. The time discretization of this convolution operator is then very delicate to achieve since an unsuitable discretization may destroy the underlying stability of the interior Crank-Nicolson scheme [24] and lead to an ill-posed problem. Several solutions have been considered to remedy to this problem [1], 4], 6], 7], 8], 11, [13], [14, [26], 27].

Studies concerning the construction of the exact boundary condition in two dimensions have received less attention, and only a few developments have recently been achieved. To the best of the authors' knowledge, the analysis has been restricted only to some canonical geometries such as the half-plane [12], 13, [20], 23] or the circular (and cylindrical) cases 19], 17, 25] since the construction can be developed (as in the one-dimensional case) by the classical Fourier or Laplace analysis. Unfortunately, these conditions appear to be non-local both in space and time and hence lead to a prohibitive computational cost even if it can be reduced with the help of a fast evaluation algorithm (see, e.g., [23], 25]). A prospective direction has been the design of some fully localized approximate boundary conditions (also called artificial boundary conditions) involving only some differential operators [1], [12, [13], [20]. Even if these conditions are efficient, they may generate some unphysical reflections at the boundary which can be due, for instance, to the presence of singularities in the geometry of the domain (generally a rectangular domain). Moreover, there are only a few results concerning the well-posedness of the resulting truncated initial boundary value problems. We have recently proposed in 3 an alternative approach to the ones cited above which allows us to construct a hierarchy of artificial boundary conditions for an arbitrarily shaped boundary. These conditions are non-local in time but present the interesting feature of being local in space, this point being essential from a practical point of view since the approximation of the problem by a finite-difference or finite-element method leads to the resolution of a linear system defined by a sparse matrix. Therefore, in Section 2 we propose a brief review of the main results stated in [3]. Next, we study the truncated initial boundary value problem with one of the previous artificial boundary conditions. The uniqueness of the solution is then proved as well as the decay of the total energy associated to the system. In the third section, we investigate the wellposedness of the semi-discrete problem discretized by the Crank-Nicolson scheme. The fractional time operators arising in the definition of the artificial boundary conditions are approximated by some quadrature formulas previously derived for the one-dimensional case 4 . The essential result of this section is that the decay of the energy is preserved at the semi-discrete level, implying hence the stability of the whole scheme. The two main ingredients used for the proof are the microlocal highfrequency character of the approximation of the artificial boundary conditions and the use of the $\mathcal{Z}$-transform. In a fourth section, we propose the full discretization of the system by a conforming finite-element method providing hence the stability 
of the fully discrete problem. Several numerical simulations are performed next to show the effectiveness of the approach.

\section{Artificial boundary CONDitions for the Schrödinger equation}

In this section, we briefly recall the main results concerning the construction of some artificial boundary conditions for the two-dimensional Schrödinger equation 3]. These conditions have been derived as some high-frequency microlocal approximations of the exact Dirichlet-Neumann (DN) pseudodifferential operator. They present the interesting feature of being differential in space. As a consequence, their implementation in a finite-element solver does not modify the sparse structure of the resulting linear system to solve. Moreover, they are non-local with respect to the time variable and involve some fractional derivative and integral time operators.

2.1. Artificial boundary conditions for a general surface. Let $\Omega$ be a twodimensional regular bounded computational domain with a smooth boundary $\Gamma$. We denote by $\mathbf{n}$ the outwardly directed unit normal vector to $\Omega$. Let us designate by $s$ the anticlockwise directed curvilinear abscissa along $\Gamma$ and $\kappa(s)$ the curvature at this point. We set $\Delta_{\Gamma}=\partial_{s}^{2}$ as being the Laplace-Beltrami operator. Concerning the time operators, let $\partial_{t}^{1 / 2}$ and $I_{t}^{1 / 2}$ be, respectively, the fractional derivative and integral operators of half-order [15] defined by

$$
\partial_{t}^{1 / 2} \psi(t)=\frac{1}{\sqrt{\pi}} \frac{d}{d t} \int_{0}^{t} \frac{\psi(w)}{\sqrt{t-w}} d w, \quad I_{t}^{1 / 2} \psi(t)=\frac{1}{\sqrt{\pi}} \int_{0}^{t} \frac{\psi(w)}{\sqrt{t-w}} d w .
$$

These are non-local convolution operators. Under this notation and according to [3], we have the following proposition.

Proposition 2.1. The Schrödinger equation with an artificial boundary condition of DN type of order $m / 2$, with $m \in\{1, \ldots, 4\}$, is defined by the initial boundary value problem

$$
\left(D N^{m / 2}\right)\left\{\begin{array}{l}
\left(i \partial_{t}+\Delta\right) u=0, \quad(x, t) \in \Omega \times \mathbb{R}^{*+} \\
\partial_{\mathbf{n}} u+T_{m / 2} u=0, \quad(x, t) \in \Gamma \times \mathbb{R}^{*+}, \\
u(x, 0)=u_{0}(x), \quad x \in \Omega,
\end{array}\right.
$$

where $T_{m / 2}, m \in\{1, \ldots, 4\}$, are pseudodifferential in time and differential in space operators given by

$$
\begin{aligned}
& T_{1 / 2} u=e^{-i \pi / 4} \partial_{t}^{1 / 2} u, \quad T_{1} u=T_{1 / 2} u+\frac{\kappa}{2} u, \quad \text { on } \Gamma \times \mathbb{R}^{*+}, \\
& T_{3 / 2} u=T_{1} u-e^{i \pi / 4}\left(\frac{\kappa^{2}}{8}+\frac{1}{2} \Delta_{\Gamma}\right) I_{t}^{1 / 2} u, \quad \text { on } \Gamma \times \mathbb{R}^{*+}, \\
& T_{2} u=T_{3 / 2} u+i\left(\frac{\kappa^{3}}{8}+\frac{1}{2} \partial_{s}\left(\kappa \partial_{s}\right)+\frac{\Delta_{\Gamma} \kappa}{8}\right) I_{t} u, \quad \text { on } \Gamma \times \mathbb{R}^{*+} .
\end{aligned}
$$

These conditions (which are symmetrical in the sense of $L^{2}(\Gamma)$ ) are constructed in the high-frequency regime. This is the essential point of all the construction of the above artificial boundary conditions developed in [3. More precisely, let us denote by $\xi$ and $\tau$ the respective covariables of $s$ and $t$ by the Fourier transform. Following [3], we introduce the $M$ quasi-hyperbolic zone as the set $\mathcal{H}$ of points $(s, t, \xi, \tau)$ defined by

$$
\mathcal{H}=\left\{(s, t, \xi, \tau), \tau+\xi^{2}<0\right\}
$$




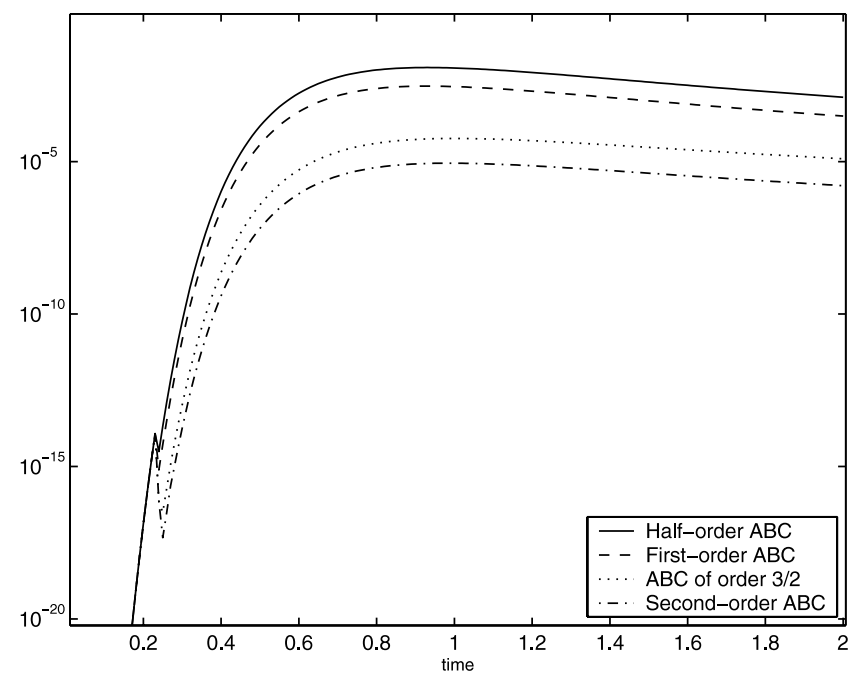

Figure 1. Time evolution of the error between the exact and approximate normal derivatives at the point $(-10,0)$ for a gaussian distribution propagating in $D(0,10)$.

The pair $M=(1,2)$ makes precise the inhomogeneities present between the $\xi$ and $\tau$ dual variables. The construction of the artificial boundary conditions is then developed under the (microlocal) assumption that the points $(s, t, \xi, \tau)$ are in $\mathcal{H}$. They characterize the propagative part of the wave $u$. Two other regions can be also defined: the $M$ quasi-elliptic region $\mathcal{E}$ given by

$$
\mathcal{E}=\left\{(s, t, \xi, \tau), \tau+\xi^{2}>0\right\},
$$

which corresponds to the evanescent (exponentially decreasing) part of $u$ and the $M$ quasi-glancing zone which is the complementary set $\mathcal{G}$ of $\mathcal{E} \cup \mathcal{H}$. This last region is reduced to $\{0\}$ if the wave $u$ is not tangentially incident to $\Gamma$. At this point of the paper, we always assume that the frequencies are defined in the quasi-hyperbolic zone $\mathcal{H}$. This assumption is not always valid but it is fulfilled if we assume that the evanescent part of the wave has vanished and hence that $\mathcal{E}$ is also reduced to $\{0\}$. This assumption is numerically illustrated in Figure 1 where we can see that the exact operator is well approximated.

To show the efficiency of these conditions from a continuous point of view, we can compute the theoretical error between the normal derivative trace of the exact solution $\partial_{\mathbf{n}} u_{\mid \Gamma}$ to the initial boundary value problem (1.1) and the approximate normal derivative trace computed by $-T_{m / 2} u$. To this end, we plot (using a symbolic computer algebra system) on Figure 1 the time evolution of $\partial_{\mathbf{n}} u+T_{m / 2} u$ in logarithmic scale at the boundary point $(-10,0)$ for a gaussian initial datum

$$
u_{0}(x)=e^{-5 i x_{1}-\left(x_{1}^{2}+x_{2}^{2}\right)},
$$

travelling in the $(-1,0)$ direction in the disk $D(0,10)$ (centred at the origin and of radius $R=10$ ). This point has been chosen since it generally represents a realistic upper bound to the reflection observed at any other point, the wave being of maximal magnitude at this point. In the ideal case (by considering instead the 
exact condition), this difference would vanish. Here, some small spurious reflections occur. As can be observed, increasing the order of the artificial boundary condition results in enhancement of the accuracy. This indicates that these conditions yield very satisfactory approximations and, moreover, that the second-order artificial boundary condition gives the most accurate results. This also confirms that the high-frequency hypothesis is satisfied: the frequencies are defined in the $M$ quasihyperbolic zone $\mathcal{H}$.

Using the pseudo-inverse operator of the DN operator, we can also derive some artificial conditions of DN type. This may be useful for the development of dual formulations for mixed finite element approximations (which are not investigated here), those based on the DN operator being more suitable for a primal weak formulation (the point of view adopted in Section (4). To illustrate this remark, we only give the artificial boundary condition of the DN type of order $3 / 2$ :

$$
u+\left(e^{i \pi / 4} I_{t}^{1 / 2}-\frac{i \kappa}{2} I_{t}+e^{-3 i \pi / 4}\left(\frac{1}{2} \Delta_{\Gamma}+\frac{3}{8} \kappa^{2}\right) I_{t}^{3 / 2}\right) \partial_{\mathbf{n}} u=0 \text { on } \Gamma \times \mathbb{R}^{*+},
$$

where $I_{t}^{\alpha}$ is the fractional integral time operator of order $\alpha$ [15].

2.2. Study of the truncated initial boundary value problem. Let us consider the Schrödinger equation set in the whole-space of propagation with an initial condition

$$
\begin{cases}\left(i \partial_{t}+\Delta\right) u=0, & (x, t) \in \mathbb{R}^{2} \times \mathbb{R}^{*+} \\ u(x, 0)=u_{0}(x), & \forall x \in \mathbb{R}^{2}\end{cases}
$$

For reasons linked to the numerical approximation by a finite-difference scheme in the time domain, the study is restricted to the case of a bounded time interval $[0, T]$, where the maximal time $T$ is supposed to be given. A classical result then gives the existence and uniqueness of the solution to the initial boundary value problem (2.6) and the conservation with respect to the time of the associated energy in $L^{2}\left(\mathbb{R}^{2}\right)$ norm. Here, the space of square integrable functions $L^{2}(D)$ is equipped with the norm $\|\cdot\|_{0}$ if $D=\mathbb{R}^{2}$ or $\|\cdot\|_{0, \Omega}$ if $D=\Omega$. More precisely, the following result holds.

Proposition 2.2. If the initial datum satisfies $u_{0} \in L^{2}\left(\mathbb{R}^{2}\right)$, then the initial boundary value problem (2.6) admits one and only one solution $u \in \mathcal{C}\left([0, T], L^{2}\left(\mathbb{R}^{2}\right)\right)$. Furthermore, we have the time conservation of the total energy of the system

$$
\|u(t)\|_{0}=\left\|u_{0}\right\|_{0} \quad \forall t \in[0, T] .
$$

If $u$ is restricted to $\Omega$ and if we assume that the initial datum $u_{0}$ is compactly supported in $\Omega$, this equality becomes an inequality which yields the decay of the energy on $\Omega$. This property has to be preserved for the truncated initial boundary value problem with an artificial boundary condition, this one being often called an absorbing boundary condition. The result is embedded in the following proposition.

Proposition 2.3. Let us assume that the assumption of high frequency is satisfied for the wave $u$ and the fictive boundary $\Gamma$. If the initial datum satisfies $u_{0} \in L^{2}(\Omega)$ and if $u$ is solution on $[0, T]$ to the initial boundary value problem (2.2), then $u$ is unique and is defined in $\mathcal{C}\left([0, T], L^{2}(\Omega)\right)$. Moreover, the solution fulfils the inequality

$$
\|u(t)\|_{0, \Omega} \leq\left\|u_{0}\right\|_{0, \Omega} \quad \forall t \in[0, T] .
$$


Proof. The proof is only detailed for the second-order artificial boundary condition $\left(D N^{2}\right)$, the involvement of any other condition of lower order being immediately deduced from the following proof. Let us prove that the decay of the energy is satisfied for any time $\tilde{T} \in] 0, T]$. Let $\tilde{u}(., t)$ be the function equal to $u(., t)$ for $t \in[0, \tilde{T}]$ and extended by 0 for $\mathbb{R} \backslash[0, \tilde{T}]$. Hence, in the distribution sense, we have the equations

- $\partial_{t} \tilde{u}(x, t)=\left\{\begin{array}{l}\partial_{t} u(x, t)-u(x, \tilde{T}) \delta_{\tilde{T}}(t)+u(x, 0) \delta_{0}(t), \quad t \in[0, \tilde{T}] \\ 0, \quad t \in]-\infty, 0[\cup] \tilde{T},+\infty[\end{array}\right.$

- $\Delta \tilde{u}(x, t)=\left\{\begin{array}{l}\Delta u(x, t), \quad t \in[0, \tilde{T}], \\ 0, \quad t \in]-\infty, 0[\cup] \tilde{T},+\infty[,\end{array}\right.$

- $\partial_{\mathbf{n}} \tilde{u}+T_{2} \tilde{u}=0, \quad(x, t) \in \Gamma \times \mathbb{R}$.

Then system (2.2) may be rewritten as

$$
\left\{\begin{array}{l}
\left(i \partial_{t}+\Delta\right) \tilde{u}(x, t)=-i u(x, \tilde{T}) \delta_{\tilde{T}}(t)+i u(x, 0) \delta_{0}(t), \quad(x, t) \in \Omega \times \mathbb{R}, \\
\partial_{\mathbf{n}} \tilde{u}(x, t)+T_{2} \tilde{u}(x, t)=0, \quad(x, t) \in \Gamma \times \mathbb{R}, \\
\tilde{u}(x, 0)=u_{0}(x), \quad x \in \Omega .
\end{array}\right.
$$

Multiplying the Schrödinger equation appearing in (2.8) by $-i \overline{\tilde{u}(x, t)}$ and next using the Green formula on $\Omega$, we get the weak variational equation

$$
\begin{aligned}
\int_{\Omega} \partial_{t} \tilde{u}(x, t) \overline{\tilde{u}(x, t)} d \Omega & -i \int_{\Gamma} \partial_{\mathbf{n}} \tilde{u}(x, t) \overline{\tilde{u}(x, t)} d \Gamma+i\|\nabla \tilde{u}(t)\|_{0, \Omega}^{2} \\
& =-\int_{\Omega} u(x, \tilde{T}) \overline{\tilde{u}(x, t)} \delta_{\tilde{T}}(t) d \Omega+\int_{\Omega} u_{0}(x) \delta_{0}(t) \overline{\tilde{u}(x, t)} d \Omega .
\end{aligned}
$$

Taking the real part of the above equation, integrating according to the time $t$ on $\mathbb{R}$, remarking that $\tilde{u}(x, t= \pm \infty)=0$ and that $\|\tilde{u}(t)\|_{0, \Omega}=\|u(t)\|_{0, \Omega}, \forall t \in[0, \tilde{T}]$, we deduce the equality

$$
\begin{aligned}
\|u(\tilde{T})\|_{0, \Omega}^{2}-\left\|u_{0}\right\|_{0, \Omega}^{2}=2 \Re\left(i \int_{\Gamma \times \mathbb{R}} \partial_{\mathbf{n}} \tilde{u}\left(x, t^{\prime}\right) \overline{\tilde{u}\left(x, t^{\prime}\right)} d \Gamma d t^{\prime}\right) \\
=-2 \Re\left(i \int_{\Gamma \times \mathbb{R}} T_{2} \tilde{u}\left(x, t^{\prime}\right) \overline{\tilde{u}\left(x, t^{\prime}\right)} d \Gamma d t^{\prime}\right) .
\end{aligned}
$$

Hence, if we prove that the quantity appearing in the right-hand side of the above equation is negative, then we have proved the result of the proposition.

Let us consider the following splitting of the artificial boundary condition into five terms:

$$
\begin{aligned}
& 2 \Re\left(i \int_{\Gamma \times \mathbb{R}} T_{2} \tilde{u}\left(x, t^{\prime}\right) \overline{\tilde{u}\left(x, t^{\prime}\right)} d \Gamma d t^{\prime}\right)=\Re\left(i \int_{\Gamma \times \mathbb{R}} \kappa\left|\tilde{u}\left(x, t^{\prime}\right)\right|^{2} d \Gamma d t^{\prime}\right) \\
& \quad+\Re\left(\int_{\Gamma \times \mathbb{R}} \frac{\kappa^{2}}{4} e^{-i \pi / 4} I_{t^{\prime}}^{1 / 2} \tilde{u}\left(x, t^{\prime}\right) \overline{\tilde{u}}\left(x, t^{\prime}\right) d \Gamma d t^{\prime}\right) \\
& \quad+\Re\left(\int_{\Gamma \times \mathbb{R}} i\left(2 e^{-i \pi / 4} \partial_{t^{\prime}}^{1 / 2} \tilde{u}\left(x, t^{\prime}\right)-e^{i \pi / 4} \Delta_{\Gamma} I_{t^{\prime}}^{1 / 2} \tilde{u}\left(x, t^{\prime}\right)\right) \overline{\tilde{u}\left(x, t^{\prime}\right)} d \Gamma d t^{\prime}\right) \\
& \quad-\Re\left(\int_{\Gamma \times \mathbb{R}}\left(\frac{\kappa^{3}}{4}+\frac{\Delta_{\Gamma} \kappa}{4}\right) I_{t^{\prime}} \tilde{u}\left(x, t^{\prime}\right) \overline{\tilde{u}\left(x, t^{\prime}\right)} d \Gamma d t^{\prime}\right) \\
& \quad+\Re\left(\int_{\Gamma \times \mathbb{R}} \kappa I_{t^{\prime}} \partial_{s} \tilde{u}\left(x, t^{\prime}\right) \partial_{s} \overline{\tilde{u}\left(x, t^{\prime}\right)} d \Gamma d t^{\prime}\right) .
\end{aligned}
$$


The first term of the right-hand side vanishes and the second one is treated using the fact that the half-order integral operator is positive in the sense of operators with memory [5], [4].

Lemma 2.4. Let $f \in H^{-1 / 4}(0, t)$, then function $\tilde{f}$, extension of $f$ by 0 on ]$-\infty, 0[\cup] t,+\infty[$, satisfies the inequality

$$
\Re\left(\int_{\mathbb{R}} e^{-i \frac{\pi}{4}} I_{t^{\prime}}^{1 / 2} \tilde{f}\left(t^{\prime}\right) \overline{\tilde{f}\left(t^{\prime}\right)} d t^{\prime}\right) \geq 0 .
$$

The determination of the sign of the third term is more delicate and is obtained as follows. Let us recall that $\tau$ and $\xi$ designate the respective covariables of $t^{\prime}$ and $s$ by the Fourier transform and let $\widehat{\tilde{u}}$ be the Fourier transform of $\tilde{u}$. The Plancherel theorem implies that we have

$$
\begin{aligned}
& \Re\left(\int_{\Gamma \times \mathbb{R}} i\left(2 e^{-i \pi / 4} \partial_{t^{\prime}}^{1 / 2} \tilde{u}\left(x, t^{\prime}\right)-e^{i \pi / 4} \Delta_{\Gamma} I_{t^{\prime}}^{1 / 2} \tilde{u}\left(x, t^{\prime}\right)\right) \overline{\tilde{u}\left(x, t^{\prime}\right)} d \Gamma d t^{\prime}\right) \\
& =\int_{\mathbb{R} \times \mathbb{R}} \Re\left(A(\xi, \tau)|\widehat{\tilde{u}}(\xi, \tau)|^{2}\right) d \xi d \tau,
\end{aligned}
$$

where symbol $A$ is defined by

$$
A(\xi, \tau)=i\left(2 e^{-i \pi / 4} \sqrt{i \tau}+e^{i \pi / 4} \frac{\xi^{2}}{\sqrt{i \tau}}\right) .
$$

Let us study the sign of $A$ with respect to $\xi$ and $\tau$. We recall that the approximation is microlocally developed under the assumption that the frequencies belong to the $M$ quasi-hyperbolic zone $\tau+\xi^{2}<0$. Therefore, the following two cases appear:

- If $\tau>0$, we may write $A$ as $A(\xi, \tau)=i\left(2 \sqrt{\tau}+\xi^{2} / \sqrt{\tau}\right)$, and then we have $\Re(A(\xi, \tau))=0$.

- If $\tau<0$, then $A(\xi, \tau)=2 \sqrt{-\tau}-\xi^{2} / \sqrt{-\tau}$, but since the frequencies are defined in the anisotropic cone $\mathcal{H}$, we can write that $\sqrt{-\tau}-\xi^{2} / \sqrt{-\tau}>0$. As a consequence, we obtain $A(\xi, \tau)>\sqrt{-\tau} \geq 0$.

From this study, we can see that the third term of (2.9) is negative. Finally, the last two terms vanish if we use the Plancherel theorem on the time variable. This ends the proof concerning the decay of the energy (2.7). Uniqueness is simply a consequence of this inequality.

\section{Stable Semi-DisCRETE CRANK-NiCOLSON-TyPe SChEMES FOR THE SCHRÖDINGER EQUATION \\ WITH A HIGH-FREQUENCY ARTIFICIAL BOUNDARY CONDITION}

A well-known, second-order and stable numerical scheme to approximate the linear Schrödinger equation in the whole-space (2.6) is given by the implicit CrankNicolson scheme. However, when the spatial domain is truncated by the introduction of a fictive boundary, an unsuitable discretization of the artificial boundary condition (and, more specifically, of time-fractional operators) can lead to loss of stability of the interior scheme (see, e.g., Mayfield [24]). For this reason, several works have been devoted to solving this problem in the one-dimensional case [4] [7, [8, 11], [14], [27]. We propose here an unconditionally stable time semidiscretization of the truncated boundary value problem (2.2) for the Crank-Nicolson scheme. It is based on some previous results developed in the one-dimensional case 
[4] using the principle of images [16] for the discretization of the fractional operators. After having recalled these results, we construct the semi-discrete scheme associated to (2.2). To prove the stability of the proposed scheme, we make some recalls on the $\mathcal{Z}$-transform of a signal which will be often used in the rest of this paper. Then we get the semi-discrete version of Proposition 2.3

3.1. Preliminary results. In the rest of this paper if $\delta t$ designates a time step, $t_{n}=n \delta t$ stands for the $n$-th time step, where $n \in \mathbb{N}$. We denote by $u^{n}$ an approximate value of $u$ at time $t_{n}$. If we consider the one-dimensional Schrödinger equation, one can prove that the exact and artificial boundary conditions are the same. Then we have the representation of the normal derivative trace as a function of the trace by the relation

$$
\partial_{\mathbf{n}} u=-e^{-i \pi / 4} \partial_{t}^{1 / 2} u, \quad(x, t) \in \Gamma \times \mathbb{R}^{*+},
$$

where $\Gamma$ represents a point of $\mathbb{R}$. If we consider the Schrödinger equation set on an interval $] a, b\left[\right.$, associated to an initial condition $u_{0}$ such that $\left.\operatorname{Supp}\left(u_{0}\right) \subset\right] a, b[$ and imposing the boundary condition (3.1) on $\Gamma=\{a, b\}$, it is possible to design some suitable quadrature schemes for the operators $I_{t}^{1 / 2}$ and $\partial_{t}^{1 / 2}$ which allow us to preserve the stability of the semi-discrete Crank-Nicolson scheme [4], 14].

Proposition 3.1. If $\left\{f_{n}\right\}_{n \in \mathbb{N}}$ designates a sequence of complex values approximating $\left\{f\left(t_{n}\right)\right\}_{n \in \mathbb{N}}$, then the approximations of $\partial_{t}^{1 / 2} f\left(t_{n}\right)$ and $I_{t}^{1 / 2} f\left(t_{n}\right)$ are given by the numerical quadrature formulas

$$
I_{t}^{1 / 2} f\left(t_{n}\right) \approx \frac{\sqrt{2 \delta t}}{2} \sum_{k=0}^{n} \alpha_{k} f^{n-k}
$$

and

$$
\partial_{t}^{1 / 2} f\left(t_{n}\right) \approx \frac{2}{\sqrt{2 \delta t}} \sum_{k=0}^{n} \beta_{k} f^{n-k}
$$

where $\left(\alpha_{k}\right)_{k \in \mathbb{N}}$ and $\left(\beta_{k}\right)_{k \in \mathbb{N}}$ designate the sequences defined by

$$
\left\{\begin{array}{l}
\left(\alpha_{0}, \alpha_{1}, \alpha_{2}, \alpha_{3}, \alpha_{4}, \alpha_{5}, \cdots\right)=\left(1,1, \frac{1}{2}, \frac{1}{2}, \frac{3}{8}, \frac{3}{8}, \cdots\right), \\
\beta_{k}=(-1)^{k} \alpha_{k} \quad \forall k \geq 0 .
\end{array}\right.
$$

In fact, the above discretizations correspond to the fractional trapezoidal rules given, for instance, in [22]. This is finally quite natural since the Crank-Nicolson scheme is derived from the usual trapezoidal quadrature formula.

To have a more concise writing of the schemes developed below, we will often use the discrete convolution $\star$ of two complex sequences $\left(f_{n}\right)_{n \in \mathbb{N}}$ and $\left(g^{n}\right)_{n \in \mathbb{N}}$ :

$$
\left(f_{n} \star g^{n}\right)_{n}=\sum_{k=0}^{n} f_{k} g^{n-k} .
$$

The proof of some results will require the use of the $\mathcal{Z}$-transform of a signal and some of its standard properties [29. Let us recall that if $\left(f_{n}\right)_{n \in \mathbb{N}}$ is a given sequence of complex values, we denote by $\mathcal{Z}\left(f_{n}\right)$ the complex-valued function defined for $|z|>R\left(\mathcal{Z}\left(f_{n}\right)\right)$ by

$$
\mathcal{Z}\left(f_{n}\right)(z)=\sum_{n=0}^{+\infty} f_{n} z^{-n},
$$


where $R\left(\mathcal{Z}\left(f_{n}\right)\right)$ is the radius of convergence of the Legendre series $\mathcal{Z}\left(f_{n}\right)$. According to this definition, one can prove the property of summation of two delayed sequences

$$
\mathcal{Z}\left(f_{n+1} \pm f_{n}\right)(z)=(z \pm 1) \mathcal{Z}\left(f_{n}\right)(z)-z f_{0} .
$$

For two singular parts of the Legendre series $\mathcal{Z}\left(f_{n}\right)$ and $\mathcal{Z}\left(g^{n}\right)$ defined for $|z|>$ $R\left(\mathcal{Z}\left(f_{n}\right)\right)$ and $|z|>R\left(\mathcal{Z}\left(g^{n}\right)\right)$, respectively, we can introduce the $\mathcal{Z}$-transform of the discrete convolution $f_{n} \star g^{n}$. More precisely, if $|z|>\max \left(R\left(\mathcal{Z}\left(f_{n}\right)\right), R\left(\mathcal{Z}\left(g^{n}\right)\right)\right)$, then the formula

$$
\mathcal{Z}\left(f_{n} \star g^{n}\right)=\mathcal{Z}\left(f_{n}\right) \mathcal{Z}\left(g^{n}\right)
$$

holds. Finally, we recall the Plancherel theorem for the $\mathcal{Z}$-transform.

Lemma 3.2. Let $\mathcal{Z}\left(f_{n}\right)$ and $\mathcal{Z}\left(g^{n}\right)$ be two $\mathcal{Z}$-transforms defined for $|z|>R\left(\mathcal{Z}\left(f_{n}\right)\right)$ and $|z|>R\left(\mathcal{Z}\left(g^{n}\right)\right)$, respectively. Then, if $R\left(\mathcal{Z}\left(f_{n}\right)\right)<1$ and $R\left(\mathcal{Z}\left(g^{n}\right)\right)<1$, we have the result

$$
\sum_{n=0}^{\infty} f_{n} \overline{g^{n}}=\frac{1}{2 \pi} \int_{0}^{2 \pi} \mathcal{Z}\left(f_{n}\right)\left(e^{i \varphi}\right) \overline{\mathcal{Z}\left(g^{n}\right)\left(e^{i \varphi}\right)} d \varphi
$$

To these few properties about the $\mathcal{Z}$-transform we add some useful results on the coefficients $\alpha_{n}$ and $\beta_{n}$ into the following lemma [4].

Lemma 3.3. Let $\left(\alpha_{n}\right)_{n \in \mathbb{N}}$ and $\left(\beta_{n}\right)_{n \in \mathbb{N}}$ be the two sequences introduced in Proposition 3.1. Then, for all complex numbers $z$ with $|z|>1$, we have the two expressions

$$
\mathcal{Z}\left(\alpha_{n}\right)(z)=i \sqrt{\frac{1+z}{1-z}} \quad \text { and } \quad \mathcal{Z}\left(\beta_{n}\right)(z)=-i \sqrt{\frac{1-z}{1+z}} .
$$

Using these results, we can establish a quadrature formula for the integral $I_{t}$ which is consistent with the approximations of $I_{t}^{1 / 2}$ and $\partial_{t}^{1 / 2}$, given previously in Proposition 3.1 .

Proposition 3.4. For each integer $n \in \mathbb{N}$, let $f_{n}$ be an approximation of $f\left(t_{n}\right)$. A numerical quadrature formula for $I_{t} f\left(t_{n}\right)$ is then given by

$$
I_{t} f\left(t_{n}\right) \approx \frac{\delta t}{2} \sum_{k=0}^{n} \delta_{k} f^{n-k}
$$

where $\left(\delta_{k}\right)_{k \in \mathbb{N}}$ is the sequence defined by $\left(\delta_{0}, \delta_{1}, \delta_{2}, \delta_{3}, \ldots\right)=(1,2,2,2, \ldots)$. Moreover, the $\mathcal{Z}$-transform of the sequence $\left(\delta_{k}\right)_{k \in \mathbb{N}}$ is given by

$$
\mathcal{Z}\left(\delta_{n}\right)(z)=-\frac{1+z}{1-z}
$$

for any complex number $z$ fulfilling $|z|>1$.

Remark 3.5. For an initial datum vanishing on $\Gamma$, the quadrature formula (3.7) exactly coincides with the trapezoidal rule. This is in fact quite natural since the Crank-Nicolson scheme is derived from this last quadrature rule. This is coherent with formula (3.2) for the fractional integral operator. Furthermore, formulas (3.2), (3.3), and (3.7) satisfy the analogous semi-discrete versions of continuous operational relations of composition: $\partial_{t}^{1 / 2} I_{t}=I_{t} \partial_{t}^{1 / 2}=I_{t}^{1 / 2}$. By recursivity and using the $\mathcal{Z}$-transform, we can even deduce from a discrete point of view that we also have $I_{t}^{\alpha / 2}=\left(I_{t}^{1 / 2}\right)^{\alpha}$, for $\alpha \in \mathbb{N}$. This relation can be useful for increasing the 
order of the DN artificial boundary conditions or for designing the semi-discrete schemes associated to the DN artificial boundary conditions (2.5).

3.2. Stable semi-discrete Crank-Nicolson-type schemes. As mentioned above, the interior scheme used is the Crank-Nicolson scheme. To define the semidiscrete scheme, we introduce the following notation:

$$
\begin{aligned}
& u^{n+\frac{1}{2}}=\frac{u^{n+1}+u^{n}}{2}, \quad a^{n+\frac{1}{2}}=\frac{\beta_{n+1} \star u^{n+1}+\beta_{n} \star u^{n}}{2}, \\
& b_{\gamma}^{n+\frac{1}{2}}=\frac{\alpha_{n+1} \star \partial_{s}^{\gamma} u^{n+1}+\alpha_{n} \star \partial_{s}^{\gamma} u^{n}}{2}, \quad \gamma \in\{0,1,2\},
\end{aligned}
$$

and

$$
d_{\gamma}^{n+\frac{1}{2}}=\frac{\delta_{n+1} \star \partial_{s}^{\gamma} u^{n+1}+\delta_{n} \star \partial_{s}^{\gamma} u^{n}}{2}, \gamma \in\{0,1\} .
$$

In the rest of this paper, we denote by $S D^{m / 2}, m \in\{1, \ldots, 4\}$, the time semi-discrete version of the initial boundary value problem (2.2) given for $n \in[0, N]$ by

$$
\left(S D^{m / 2}\right)\left\{\begin{array}{l}
i \frac{u^{n+1}-u^{n}}{\delta t}+\Delta u^{n+\frac{1}{2}}=0, \quad x \in \Omega, \\
\partial_{\mathbf{n}} u^{n+\frac{1}{2}}+T_{m / 2}^{s d} u^{n+\frac{1}{2}}=0, \quad x \in \Gamma, \\
u^{0}=u_{0}, \quad x \in \Omega,
\end{array}\right.
$$

where $N$ is the number of time intervals, $N \delta t=T$. We have set $T_{m / 2}^{s d}$ as the semidiscrete approximation of the continuous artificial operator $T_{m / 2}$ by the CrankNicolson scheme. These operators are given by

$$
\begin{aligned}
& T_{1 / 2}^{s d} u^{n+\frac{1}{2}}=e^{-i \pi / 4} \sqrt{\frac{2}{\delta t}} a^{n+\frac{1}{2}}, \quad n \in[0, N], \\
& T_{1}^{s d} u^{n+\frac{1}{2}}=T_{1 / 2}^{s d} u^{n+\frac{1}{2}}+\frac{\kappa}{2} u^{n+\frac{1}{2}}, \quad n \in[0, N], \\
& T_{3 / 2}^{s d} u^{n+\frac{1}{2}}=T_{1}^{s d} u^{n+\frac{1}{2}}-e^{i \pi / 4} \sqrt{\frac{\delta t}{2}}\left(\frac{\kappa^{2} b_{0}^{n+\frac{1}{2}}}{8}-\frac{b_{2}^{n+\frac{1}{2}}}{2}\right), \quad n \in[0, N],
\end{aligned}
$$

and

$$
T_{2}^{s d} u^{n+\frac{1}{2}}=T_{3 / 2}^{s d} u^{n+\frac{1}{2}}+i \frac{\delta t}{2}\left(\left(\frac{\kappa^{3}}{8}+\frac{\Delta_{\Gamma} \kappa}{8}\right) d_{0}^{n+\frac{1}{2}}+\frac{1}{2} \partial_{s}\left(\kappa d_{1}^{n+\frac{1}{2}}\right)\right), \quad n \in[0, N] .
$$

Let us recall that the statement of the proof of Proposition 2.3 has been given under the assumption of high frequency. Here, the stability result for the semidiscrete scheme also uses a similar semi-discrete assumption. We suppose that the frequencies are some elements of a semi-discrete $M$ quasi-hyperbolic zone $\mathcal{H}^{\text {sd }}$ (see Definition 3.7). We introduce this technical notation in the proof of the proposition below.

Under the notation above, we have the analogous semi-discrete version of Proposition 2.3 for the $S D^{m / 2}$ scheme, $m \in\{1, \ldots, 4\}$.

Proposition 3.6. The schemes $S D^{m / 2}, m \in\{1, \ldots, 4\}$, are unconditionally stable under the semi-discrete high-frequency assumption (see Definition 3.7). Moreover, if $\left(u^{n}\right)_{n \in[0, N]}$ designates the solution to problem $S D^{m / 2}$, then it satisfies the energy inequality

$$
\left\|u^{n+1}\right\|_{0, \Omega} \leq\left\|u^{0}\right\|_{0, \Omega} \quad \forall n \in[0, N-1] .
$$


Proof. The proof is only given for the scheme $S D^{2}$, the other demonstrations being immediately deduced from the following one. We consider the first equation of $S D^{2}$ that we multiply by $-i \overline{u^{n+\frac{1}{2}}}$ and next integrate on the finite-computational domain $\Omega$ according to $x$. An application of the Green formula yields

$$
\begin{aligned}
\int_{\Omega} & \frac{\left|u^{n+1}\right|^{2}-\left|u^{n}\right|^{2}+2 i \Im\left(u^{n+1} \overline{u^{n}}\right)}{2 \delta t} d \Omega+i\left\|\nabla u^{n+\frac{1}{2}}\right\|_{0, \Omega}^{2} \\
= & -e^{i \pi / 4} \sqrt{\frac{2}{\delta t}} \int_{\Gamma} a^{n+\frac{1}{2}} \overline{u^{n+\frac{1}{2}}} d \Gamma-i \int_{\Gamma} \frac{\kappa}{2}\left|u^{n+\frac{1}{2}}\right|^{2} d \Gamma \\
& +i e^{i \pi / 4} \sqrt{\frac{\delta t}{2}}\left(\int_{\Gamma} \frac{\kappa^{2}}{8} b_{0}^{n+\frac{1}{2}} \overline{u^{n+\frac{1}{2}}} d \Gamma-\int_{\Gamma} \frac{b_{2}^{n+\frac{1}{2}}}{2} \overline{u^{n+\frac{1}{2}}} d \Gamma\right) \\
& +\frac{\delta t}{2} \int_{\Gamma}\left(\frac{\kappa^{3}}{8}+\frac{\Delta_{\Gamma} \kappa}{8}\right) d_{0}^{n+\frac{1}{2}} \overline{u^{n+\frac{1}{2}}} d \Gamma-\frac{\delta t}{4} \int_{\Gamma} \kappa d_{1}^{n+\frac{1}{2}} \partial_{s} \overline{u^{n+\frac{1}{2}}} d \Gamma
\end{aligned}
$$

Taking the real part of this last equation, summing on the indices $n \in[0, N]$ (for the sake of brevity, $N>0$ designates any integer lying in the time discretization interval), and using a few algebraic manipulations, we get

$$
\begin{aligned}
& \frac{\left\|u^{N+1}\right\|_{0, \Omega}^{2}-\left\|u^{0}\right\|_{0, \Omega}^{2}}{2 \delta t}=-\Re\left(e^{i \frac{\pi}{4}} \sqrt{\frac{1}{2 \delta t}} \sum_{n=0}^{N} \int_{\Gamma} a^{n+\frac{1}{2}} \overline{u^{n+\frac{1}{2}}} d \Gamma\right) \\
& +\Re\left(i e^{i \frac{\pi}{4}} \sqrt{\frac{\delta t}{2}} \sum_{n=0}^{N} \int_{\Gamma} \frac{\kappa^{2}}{8} b_{0}^{n+\frac{1}{2}} \overline{u^{n+\frac{1}{2}}} d \Gamma\right) \\
& +\Re\left(i e^{i \frac{\pi}{4}} \sqrt{\frac{\delta t}{2}} \sum_{n=0}^{N} \int_{\Gamma}\left(i \frac{a^{n+\frac{1}{2}}}{\delta t}-\frac{b_{2}^{n+\frac{1}{2}}}{2}\right) \overline{u^{n+\frac{1}{2}}} d \Gamma\right) \\
& +\Re\left(\frac{\delta t}{2} \sum_{n=0}^{N} \int_{\Gamma}\left(\frac{\kappa^{3}}{8}+\frac{\Delta_{\Gamma} \kappa}{8}\right) d_{0}^{n+\frac{1}{2}} \overline{u^{n+\frac{1}{2}}} d \Gamma\right)-\Re\left(\frac{\delta t}{4} \sum_{n=0}^{N} \int_{\Gamma} \kappa d_{1}^{n+\frac{1}{2}} \partial_{s} \overline{u^{n+\frac{1}{2}}} d \Gamma\right) .
\end{aligned}
$$

In order to prove the decay of the energy associated to the system, we show that each term of the right-hand side of the above equation is negative.

Let us consider the first term of the right-hand side of (3.8). To suitably use the Parseval equality (3.6), we need to extend the finite sequence $\left(u^{n}\right)_{0 \leq n \leq N}$ to an infinite sequence without modifying the quantities to estimate. To this end, we introduce the new sequence $\left(v_{n}^{N}\right)_{n \in \mathbb{N}}$ defined by

$$
v_{n}^{N}=\left\{\begin{array}{l}
u^{n}, \text { if } n \leq N+1, \\
(-1)^{k} u^{N+1}, \quad \text { if } n=N+1+k, k>0 .
\end{array}\right.
$$

We can then define the complex-valued sequences $\left(f_{n}\right)_{n}$ and $\left(g_{n}\right)_{n}$ :

$$
f_{n}=\frac{\beta_{n+1} \star v_{n+1}^{N}+\beta_{n} \star v_{n}^{N}}{2} \text { and } g_{n}=v_{n+\frac{1}{2}}^{N}=\frac{v_{n+1}^{N}+v_{n}^{N}}{2} .
$$

An immediate calculation shows that we have

$$
f_{n}=a^{n+\frac{1}{2}}, \quad \text { if } n \leq N, \quad \text { and } \quad g_{n}=\left\{\begin{array}{l}
u^{n+\frac{1}{2}}, \quad \text { if } n \leq N \\
0, \text { if } n \geq N+1 .
\end{array}\right.
$$

From the property (3.4) of the $\mathcal{Z}$-transform and since the initial datum is compactly supported, we have the relations

$$
\mathcal{Z}\left(f_{n}\right)(z)=\frac{z+1}{2} \mathcal{Z}\left(\beta_{n}\right)(z) \mathcal{Z}\left(v_{n}^{N}\right)(z) \quad \text { and } \quad \mathcal{Z}\left(g_{n}\right)(z)=\frac{z+1}{2} \mathcal{Z}\left(v_{n}^{N}\right)(z) .
$$


From the expression of $\mathcal{Z}\left(\beta_{n}\right)(z)$ given in Lemma 3.3. the Parseval formula (3.6) yields

$$
\begin{gathered}
\sum_{n=0}^{+\infty} f_{n} \overline{g_{n}}=\sum_{n=0}^{N} a^{n+\frac{1}{2}} \overline{u^{n+\frac{1}{2}}}=\frac{1}{2 \pi} \int_{[0,2 \pi[} \mathcal{Z}\left(f_{n}\right)\left(e^{i \varphi}\right) \overline{\mathcal{Z}\left(g_{n}\right)}\left(e^{i \varphi}\right) d \varphi \\
=\frac{1}{2 \pi} \int_{[0,2 \pi[}\left|\frac{e^{i \varphi}+1}{2}\right|^{2} \mathcal{Z}\left(\beta_{n}\right)\left(e^{i \varphi}\right)\left|\mathcal{Z}\left(v_{n}^{N}\right)\left(e^{i \varphi}\right)\right|^{2} d \varphi .
\end{gathered}
$$

The application $z \mapsto(1-z) /(1+z)$ being a homography which maps $D(0,1)$ onto $\{\Re(z) \geq 0\}$, we deduce that $\Re\left(e^{i \frac{\pi}{4}} \mathcal{Z}\left(\beta_{n}\right)\left(e^{i \varphi}\right)\right) \geq 0 \forall \varphi \in[0,2 \pi[$. Finally, we get

$$
\Re\left(e^{i \frac{\pi}{4}} \sqrt{\frac{2}{\delta t}} \sum_{n=0}^{N} \int_{\Gamma} a^{n+\frac{1}{2}} \overline{u^{n+\frac{1}{2}}} d \Gamma\right)=\Re\left(e^{i \frac{\pi}{4}} \sqrt{\frac{2}{\delta t}} \int_{\Gamma} \sum_{n=0}^{+\infty} f_{n} \overline{g_{n}} d \Gamma\right),
$$

providing hence the negativity of the first term.

The second term is treated as follows. Let us denote by $\widehat{u^{n}}$ the Fourier transform on $\Gamma$ of $u^{n}$ according to the curvilinear abscissa $s$, the covariable being always quoted $\xi$. Considering then the second and third terms of the right-hand side of (3.8), the Plancherel theorem yields (after an integration by parts)

$$
\begin{aligned}
& +\Re\left(i e^{i \frac{\pi}{4}} \sqrt{\frac{\delta t}{2}} \sum_{n=0}^{N} \int_{\mathbb{R}} \frac{\alpha_{n+1} \star \widehat{\kappa u^{n+1}}(\xi)+\alpha_{n} \star \widehat{\kappa u^{n}}(\xi)}{16} \widehat{\kappa u^{n+\frac{1}{2}}}(\xi) d \xi\right) \\
& +\Re\left(i e^{i \frac{\pi}{4}} \sqrt{\frac{\delta t}{2}} \sum_{n=0}^{N} \int_{\mathbb{R}}\left(i \frac{\widehat{a^{n+\frac{1}{2}}}(\xi)}{\delta t}+\xi^{2} \frac{\widehat{b_{0}^{n+\frac{1}{2}}}(\xi)}{2}\right) \widehat{\widehat{u^{n+\frac{1}{2}}}}(\xi) d \xi\right) .
\end{aligned}
$$

Let $\left(\widehat{f_{n}}\right)_{n \in \mathbb{N}}$ and $\left(\widehat{g_{n}}\right)_{n \in \mathbb{N}}$ be the Fourier transforms of the sequences $\left(f_{n}\right)_{n \in \mathbb{N}}$ and $\left(g_{n}\right)_{n \in \mathbb{N}}$, respectively, given by (3.9). Moreover, let $\left(h_{n}(\xi)\right)_{n \in \mathbb{N}}$ be the complexvalued sequence given by

$$
h_{n}(\xi)=\frac{\alpha_{n+1} \star \widehat{v_{n+1}^{N}}+\alpha_{n} \star \widehat{v_{n}^{N}}}{2} .
$$

From this last relation, we have $h_{n}=\widehat{b_{0}^{n+\frac{1}{2}}}$, if $n \leq N$. Using property (3.5) on the $\mathcal{Z}$-transform of a convolution, we deduce that

$$
\begin{aligned}
& \mathcal{Z}\left(\widehat{f_{n}}\right)(z)=\frac{z+1}{2} \mathcal{Z}\left(\beta_{n}\right)(z) \mathcal{Z}\left(\widehat{v_{n}^{N}}\right)(z), \\
& \mathcal{Z}\left(\widehat{g_{n}}\right)(z)=\frac{z+1}{2} \mathcal{Z}\left(\widehat{v_{n}^{N}}\right)(z), \\
& \mathcal{Z}\left(h_{n}\right)(z)=\frac{z+1}{2} \mathcal{Z}\left(\alpha_{n}\right)(z) \mathcal{Z}\left(\widehat{v_{n}^{N}}\right)(z),
\end{aligned}
$$

with $\mathcal{Z}\left(\beta_{n}\right)(z)$ and $\mathcal{Z}\left(\alpha_{n}\right)(z)$ given in Proposition 3.1. An application of the Parseval formula on the sequence $\left(v_{n}^{N}\right)_{n \in \mathbb{N}}$ allows us to write the first term of (3.10) as

$$
\begin{aligned}
& \Re\left(i e ^ { i \frac { \pi } { 4 } } \sqrt { \frac { \delta t } { 2 } } \sum _ { n = 0 } ^ { + \infty } \int _ { \mathbb { R } } \frac { \alpha _ { n + 1 } \star \widehat { \kappa v _ { n + 1 } ^ { N } } ( \xi ) + \alpha _ { n } \star \widehat { \kappa v _ { n } ^ { N } } ( \xi ) \widehat { \widehat { \kappa v _ { n + \frac { 1 } { 2 } } ^ { N } } } ( \xi ) d \xi ) } { 1 6 } d \xi \left(\frac{e^{i \frac{\pi}{4}}}{2 \pi} \sqrt{\frac{\delta t}{2}} \int_{[0,2 \pi[\times \mathbb{R}}\left[\frac{1}{8} \sqrt{\frac{1+z}{1-z}}\left|\frac{(z+1)}{2} \mathcal{Z}\left(\widehat{\kappa v_{n}^{N}}(\xi)\right)(z)\right|_{\mid z=e^{i \varphi}} d \varphi d \xi\right) .\right.\right.
\end{aligned}
$$


But since the application $z \mapsto(1+z) /(1-z)$ is a homography from $D(0,1)$ onto $\{\Re(z) \geq 0\}$ and which maps $C_{1}$ onto $i \mathbb{R}$, we have

$$
\arg \left(e^{i \frac{\pi}{4}}\left[\sqrt{\frac{1+z}{1-z}}\right]_{\mid z=e^{i \varphi}}\right) \in\left[0, \frac{\pi}{2}\right] .
$$

As a consequence, the quantities given by (3.11) are negative.

The treatment of the second term of (3.10) is more complicated. Let us recall that in the continuous case, the keypoint for obtaining the decay of the energy in Proposition 2.3 lies in the fact that the points $(s, t, \tau, \xi)$ belong to the $M$ quasihyperbolic zone $\mathcal{H}$. Let us try to transpose this property to the semi-discrete problem. From Definition 2.3 a quadruplet $(s, t, \xi, \tau) \in T^{*}(\Sigma)$ is in $\mathcal{H}$ if $-\tau-\xi^{2}>0$ (or equivalently $\Re\left(-\tau-\xi^{2}\right)>0$ and $\Im\left(-\tau-\xi^{2}\right)=0$ ). Let $P_{\Gamma}\left(\partial_{t}, \partial_{s}\right)$ be the pseudodifferential operator of symbol $-\tau-\xi^{2}$; it can be written as $P_{\Gamma}: f \mapsto P_{\Gamma}(f)=$ $\left(i \partial_{t}+\Delta_{\Gamma}\right) f$ and corresponds to a tangential Schrödinger equation. The semidiscrete scheme being of Crank-Nicolson type, the differential equation $\partial_{t} f(t)=g(t)$ is approximated at a point $t_{n+\frac{1}{2}}=\left(t_{n}+t_{n+1}\right) / 2$ by

$$
\frac{f\left(t_{n+1}\right)-f\left(t_{n}\right)}{\delta t}=\frac{g\left(t_{n+1}\right)+g\left(t_{n}\right)}{2} .
$$

Consequently, $P_{\Gamma}$ is approximated by the operator $\tilde{P}_{\Gamma}$ defined by

$$
\tilde{P}_{\Gamma}: f \mapsto \tilde{P}_{\Gamma}(f)=i \frac{f\left(t_{n+1}\right)-f\left(t_{n}\right)}{\delta t}+\Delta_{\Gamma} \frac{f\left(t_{n+1}\right)+f\left(t_{n}\right)}{2} .
$$

Using the Fourier transform on $\Gamma$ and the $\mathcal{Z}$-transform of the sequence $\left(f\left(t_{n}\right)\right)_{n \in \mathbb{N}}$, we then introduce the "semi-discrete symbol" of $\tilde{P}_{\Gamma}$

$$
i \frac{z-1}{\delta t}-\xi^{2} \frac{z+1}{2} \text {. }
$$

The assumption on the symbol of $P_{\Gamma}:-\tau-\xi^{2}>0$, becomes for $\tilde{P}_{\Gamma}$ at the semidiscrete level

$$
\Re\left(i \frac{z-1}{\delta t}-\xi^{2} \frac{z+1}{2}\right)>0 \text { and } \Im\left(i \frac{z-1}{\delta t}-\xi^{2} \frac{z+1}{2}\right)=0 .
$$

This leads to the following definition.

Definition 3.7. A quadruplet $(s, n, \xi, z)$ is said to be in the semi-discrete $M$ quasihyperbolic zone $\mathcal{H}^{\text {sd }}$ if it satisfies

$$
\Re\left(i \frac{z-1}{\delta t}-\xi^{2} \frac{z+1}{2}\right)>0 \text { and } \Im\left(i \frac{z-1}{\delta t}-\xi^{2} \frac{z+1}{2}\right)=0 .
$$

This analysis being done, the second term of (3.10) may be rewritten as

$$
\Re\left(i e^{i \frac{\pi}{4}} \sqrt{\frac{\delta t}{2}} \sum_{n=0}^{+\infty} \int_{\mathbb{R}}\left(i \frac{\widehat{f_{n}}(\xi)}{\delta t}+\xi^{2} \frac{\widehat{h_{n}}(\xi)}{2}\right) \widehat{\widehat{g_{n}}(\xi)} d \xi\right)
$$


or yet using the Plancherel theorem for the $\mathcal{Z}$-transform

$$
\begin{aligned}
& \Re\left(\frac{e^{i \frac{\pi}{4}}}{2 \pi} \sqrt{\frac{\delta t}{2}} \int_{[0,2 \pi[\times \mathbb{R}}\right. {\left.\left[\sigma(z)\left(i \frac{1-z}{2 \delta t}+\xi^{2} \frac{z+1}{4}\right)\left|\mathcal{Z}\left(\widehat{v_{n}^{N}}(\xi)\right)\right|^{2}\right]_{\mid z=e^{i \varphi}} d \varphi d \xi\right) } \\
&=-\int_{[0,2 \pi[\times \mathbb{R}}\left[\Re \left(\frac{e^{i \frac{\pi}{4}}}{2 \pi} \sqrt{\left.\frac{\delta t}{2} \frac{\sigma(z)}{2}\right)}\right.\right.\left.\times \Re\left(i \frac{z-1}{\delta t}-\xi^{2} \frac{z+1}{2}\right)\left|\mathcal{Z}\left(\widehat{v_{n}^{N}}(\xi)\right)\right|^{2}\right]_{\mid z=e^{i \varphi}} d \varphi d \xi \\
&+\int_{[0,2 \pi[\times \mathbb{R}}\left[\Im\left(\frac{e^{i \frac{\pi}{4}}}{2 \pi} \sqrt{\frac{\delta t}{2}} \frac{\sigma(z)}{2}\right)\right. \\
&\left.\times \Im\left(i \frac{z-1}{\delta t}-\xi^{2} \frac{z+1}{2}\right)\left|\mathcal{Z}\left(\widehat{v_{n}^{N}}(\xi)\right)\right|^{2}\right]_{\mid z=e^{i \varphi}} d \varphi d \xi,
\end{aligned}
$$

where the complex-valued function $\sigma$ is defined by

$$
\sigma(z)=\frac{\overline{z+1}}{2} \sqrt{\frac{1+z}{1-z}} .
$$

But by assumption and following Definition 3.7 the property $(s, n, \xi, z) \in \mathcal{H}^{s d}$ implies that

$$
\Re\left(i \frac{z-1}{\delta t}-\xi^{2} \frac{z+1}{2}\right)>0 \text { and } \Im\left(i \frac{z-1}{\delta t}-\xi^{2} \frac{z+1}{2}\right)=0 .
$$

Moreover, since $2 \sigma(z)=|z+1| \sqrt{1-\bar{z}^{2}} /|z-1|$ at $z=e^{i \varphi}$, we have $\arg \left(1-\bar{z}^{2}\right)$ $=-\varphi+\operatorname{sign}(\sin \varphi) \pi / 2$. As a consequence, it follows that if $\varphi \in[0,2 \pi]$, then $\arg \left(1-\bar{z}^{2}\right) \in\left[-\frac{\pi}{2}, \frac{\pi}{2}\right]$ and

$$
\Re\left(e^{i \frac{\pi}{4}} \sqrt{\frac{\delta t}{2}} \frac{\sigma(z)}{2}\right) \geq 0 .
$$

From this result, quantity (3.12) is negative and consequently so is the second term of (3.10).

Let us consider now the fourth term of the right-hand side of (3.8). By a similar approach as above, the application of the $\mathcal{Z}$-transform allows us to get the new following expression of this term by using the Parseval equality

$$
\frac{\delta t}{2} \int_{\Gamma}\left(\frac{\kappa^{3}}{8}+\frac{\Delta_{\Gamma} \kappa}{8}\right) \Re\left(\frac{1}{2 \pi} \int_{0}^{2 \pi}\left[\mathcal{Z}\left(\delta_{n}\right)\left(e^{i \varphi}\right)\left|\frac{1+e^{i \varphi}}{2}\right|^{2}\left|\mathcal{Z}\left(v_{n}^{N}\right)\left(e^{i \varphi}\right)\right|^{2}\right]_{\mid z=e^{i \varphi}} d \varphi\right) d \Gamma .
$$

But since the expression $\mathcal{Z}\left(\delta_{n}\right)(z)=-(1+z) /(1-z)$ is imaginary for $z=e^{i \varphi}$, this term vanishes. Finally, the last terms of the right-hand side of (3.8) may be treated as the previous one. Then, it is identically equal to zero.

From this analysis, we get the decay of the energy, hence ending the proof of the proposition.

Remark 3.8. All this paper is concerned with the trapezoidal rule and the associated Crank-Nicolson scheme. However, higher-order interior schemes can be developed 
to increase accuracy. This has the direct effect of modifying the numerical quadrature scheme for the convolution operator and the above proofs. However, using a similar approach should yield close results using the same kind of arguments as for the proof of stability. We refer to [22] where other kinds of discretization rules for Volterra-type operators are derived.

\section{Finite-ElEment apPROXimation And numerical RESUlts}

Finite-element approximation is based on weak formulation of the initial boundary value problem $\left(S D^{m / 2}\right)$

$$
i \int_{\Omega} \frac{u^{n+1}-u^{n}}{\delta t} \varphi d \Omega-\int_{\Gamma} T_{m / 2}^{s d} u^{n+\frac{1}{2}} \varphi d \Gamma-\int_{\Omega} \nabla u^{n+\frac{1}{2}} \cdot \nabla \varphi d \Omega=0,
$$

for any test function $\varphi \in H^{1}(\Omega)$. Since the transparent operator is of memory type, only the terms involving $u^{n+1}$ arise in the definition of the system, the other terms being put in the right-hand side of the above equation. The spatial discretization is made in the classical $\mathcal{P}_{1}$ linear finite-element space

$$
V_{h}=\left\{\varphi_{h} \in H^{1}\left(\Omega_{h}\right), \varphi_{h \mid T} \in \mathcal{P}_{1}, \forall T \in \mathcal{T}_{h}\right\},
$$

where the bounded computational polygonal domain $\Omega_{h}=\bigcup_{T \in \mathcal{T}_{h}} T$ is constructed with the help of a regular triangulation $\mathcal{T}_{h}$. The curvature approximation is developed by a simple procedure [2 based only on knowledge of the initial mesh. The finite-element approximation space $V_{h}$ being a subspace of $H^{1}\left(\Omega_{h}\right)$, the stability of the fully discrete scheme is simply a consequence of the stability of the semi-discrete scheme. The resulting complex sparse and symmetrical linear system is solved by a conjugate gradient solver accelerated by an incomplete Choleski factorization preconditioner. The convergence is reached in only a few iterations. Finally, we do not present the spatial finite-difference discretization which has been also implemented. Indeed, this approach has generally proved to be less efficient; the linear system to be solved is not symmetrical and the stability of the scheme has not been obtained.

Remark 4.1. If $N_{\Gamma}$ designates the number of boundary points, then a simple implementation of the time convolution operators would require a computational cost of about $\mathcal{O}\left(N_{\Gamma} N_{T}^{2}\right)$ operations. However, fast evaluation algorithms can be used to reduce it (this is not implemented here). This would give rise to a cost $\mathcal{O}\left(N_{\Gamma} N_{T} \log N_{T}\right)$ or $\mathcal{O}\left(N_{\Gamma} N_{T}\left(\log N_{T}\right)^{2}\right)$ using the techniques developed in [18, 22, [23], 25]. Compared for instance to the results obtained in [23, 25] which are in $\mathcal{O}\left(N_{\Gamma} N_{T} \log N_{\Gamma}\left(\log N_{T}\right)^{2}\right)$, this gives a lower complexity. However, this is at the price of a loss of accuracy since we only consider a high-frequency approximation of the exact transparent boundary condition [3].

The calculations presented are performed with the following initial datum (2.4) which (by convolution with Green's kernel) generates the time gaussian distribution

$$
u_{e x}\left(x_{1}, x_{2}, t\right)=\frac{i}{i-4 t} \exp \left(-i \frac{x_{1}^{2}+x_{2}^{2}+i k_{0} x_{1}+k_{0}^{2} i t}{i-4 t}\right) .
$$

For the linear finite-element approximation, two different computational domains are considered. The first one is the disk $D(0,2.5)$ centred at the origin (see Figure 2), and the second one is delimited by a parabola oriented along the $x_{1}$ axis and ended by a circular boundary in order to obtain a smooth curve. We take the wave number $k_{0}=5$. In the first example, the mesh is constituted of 32768 triangles whose areas 
are between $5.4 \times 10^{-4}$ and $6.4 \times 10^{-4}$, while in the second case we use 57344 triangles with an area between $5.0 \times 10^{-4}$ and 0.0108 . To have some comparable results, the time step is fixed to $\delta t=0.01$ in all computations. Even if this time step is finally not too small, some very satisfactory numerical results have been obtained. Moreover, the resulting computational complexity of the convolutions involved is not too penalizing in this case.

To appreciate the efficiency of the first- and second-order artificial boundary conditions, in Figure 4 we present the evolution of the exact reference solution at different successive time steps $t=0.25, t=0.35$, and $t=0.50$. We observe an improvement of the accuracy as the order of the artificial boundary condition increases. As can be seen in Figure 5 there are some unphysical reflections which appear when the wave strikes the left boundary. This is greatly diminished in Figure 6 for the second-order condition. Only some small reflections are present and do not pollute the propagation of the solution (the reference scale is given in Figure 3 and the error is approximately inferior to $\left.10^{-3}\right)$. This confirms that we can diminish the spurious reflection at the boundary by increasing the order of the artificial boundary condition (as it was also predicted in the case of the continuous problem in Figure 1). Moreover, this is realized without any additional cost. The half-order boundary conditions yield intermediate accuracy. To numerically fulfil the decay of the energy associated to the system, we compute the $L^{2}\left(\Omega_{h}\right)$-norm of the approximate solution in Figure 7 . The results obtained are in agreement with the predictions of Proposition 3.6. Moreover, we observe that the conditions of order $3 / 2$ and 2 yield a precise evaluation of the energy while there are some small errors for the lower-order conditions.

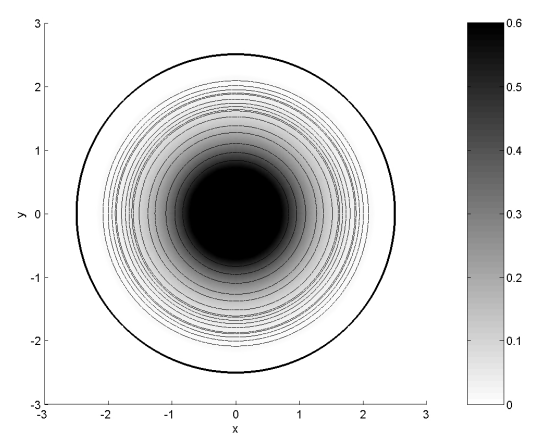

FIGURE 2. Representation of the gaussian initial distribution.

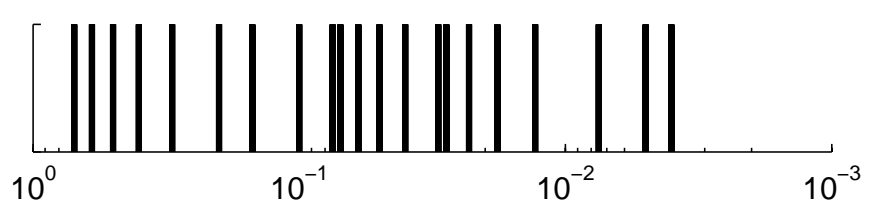

Figure 3. Level curves distribution of the wave amplitude. 
To complete the error analysis, we compute the relative error $\frac{\left\|u_{l}-u_{e x}\right\|_{2}}{\left\|u_{e x}\right\|_{2}}(t)$, where the subscript $l=1 / 2,1,3 / 2,2$ represents the order of the artificial boundary conditions. To observe the effects of the artificial boundary conditions on the dispersive behavior of the solution, we choose the wave number $k_{0}=0$ (see Figure 8). Once again, we note an improvement of the accuracy according to the order of the artificial boundary conditions. The long time error is essentially due to the artificial boundary conditions which are not exact. To display the influence of the propagative part of the solution on the error, we superpose the exact solution for
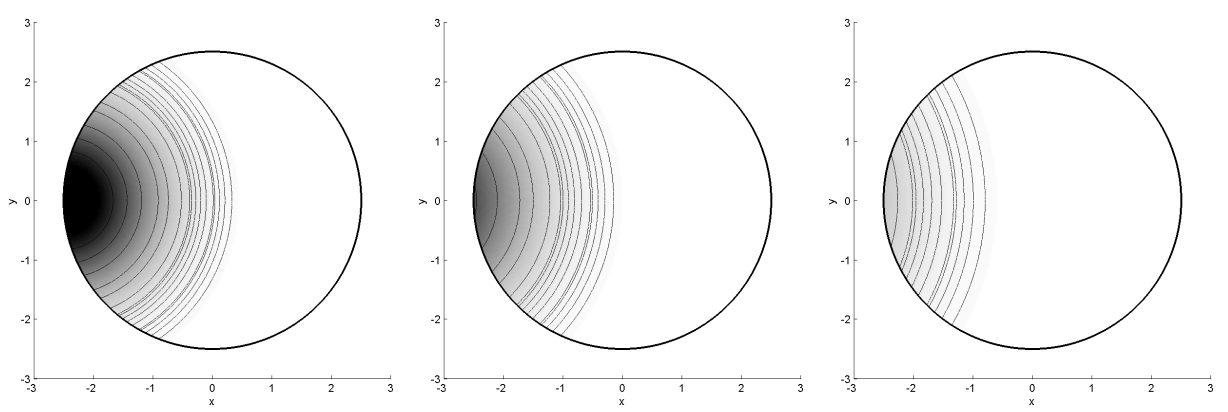

Figure 4. Evolution of the reference solution at different times: $t=0.25, t=0.35$, and $t=0.50$.
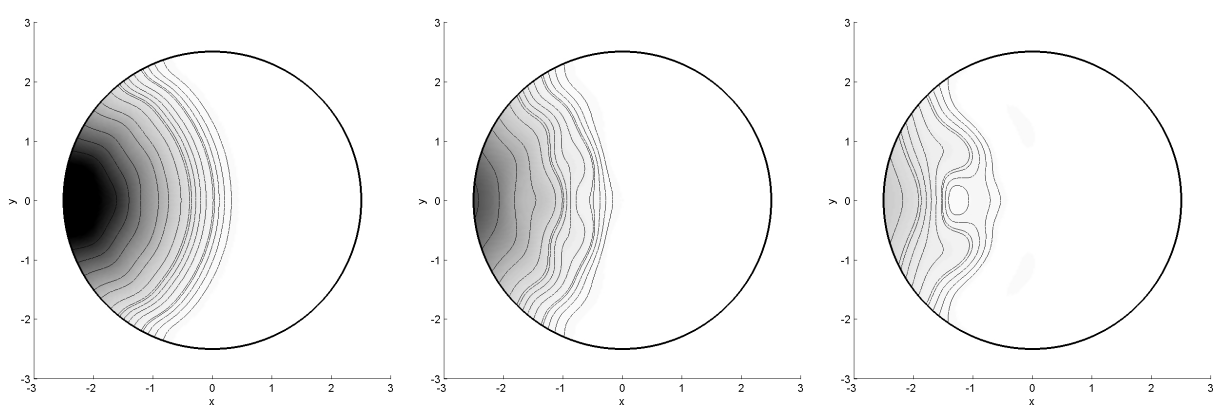

FigURE 5. Evolution of the approximate solution at different times using the first-order artificial boundary condition.
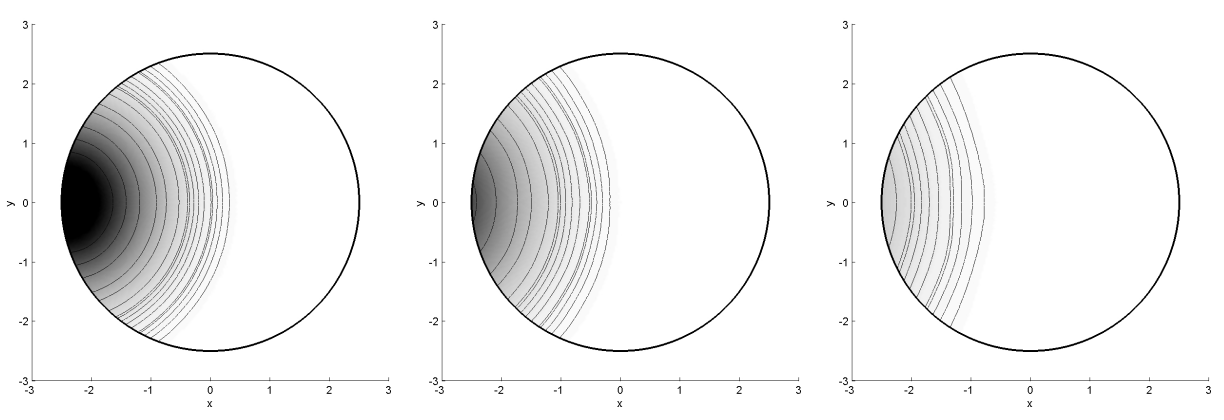

FiguRE 6. Evolution of the approximate solution at different times using the second-order artificial boundary condition. 


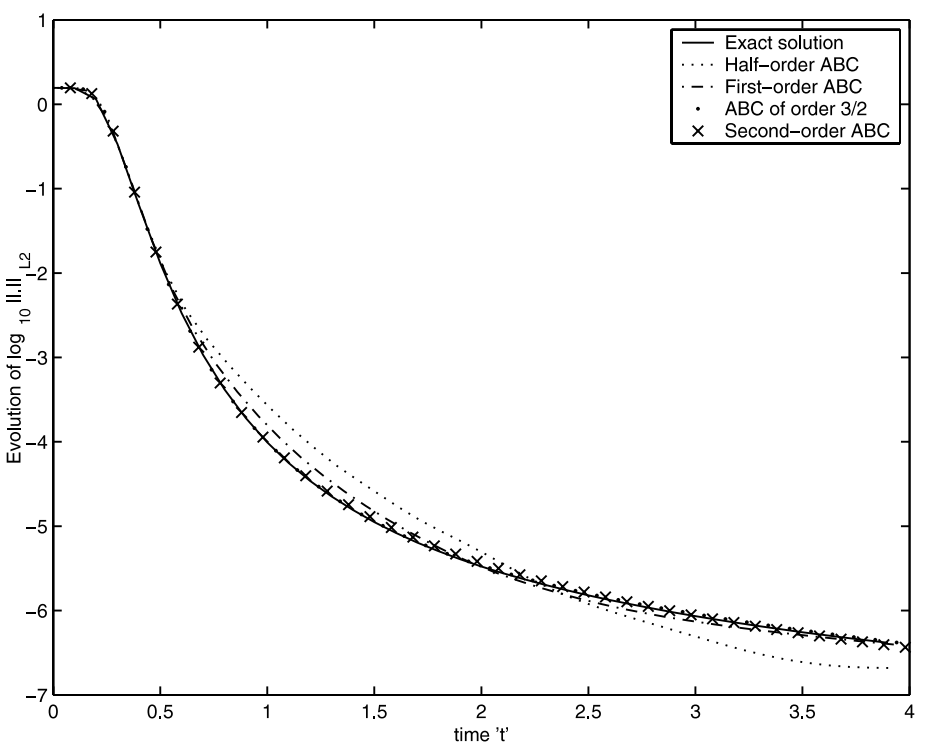

Figure 7. Evolution of $\log _{10}\|\cdot\|_{2}$ for both the exact and approximate solutions.

$k_{0}=5$ to this gaussian (see Figure 9). The maximal relative error is located at time $t \approx 0.5$ when the travelling part strikes the boundary. This bump decreases for smaller time steps. This is linked to the highly oscillating nature of the solution and to the choice of the computational discretization parameters. The long-time behavior of the relative error is not really affected.

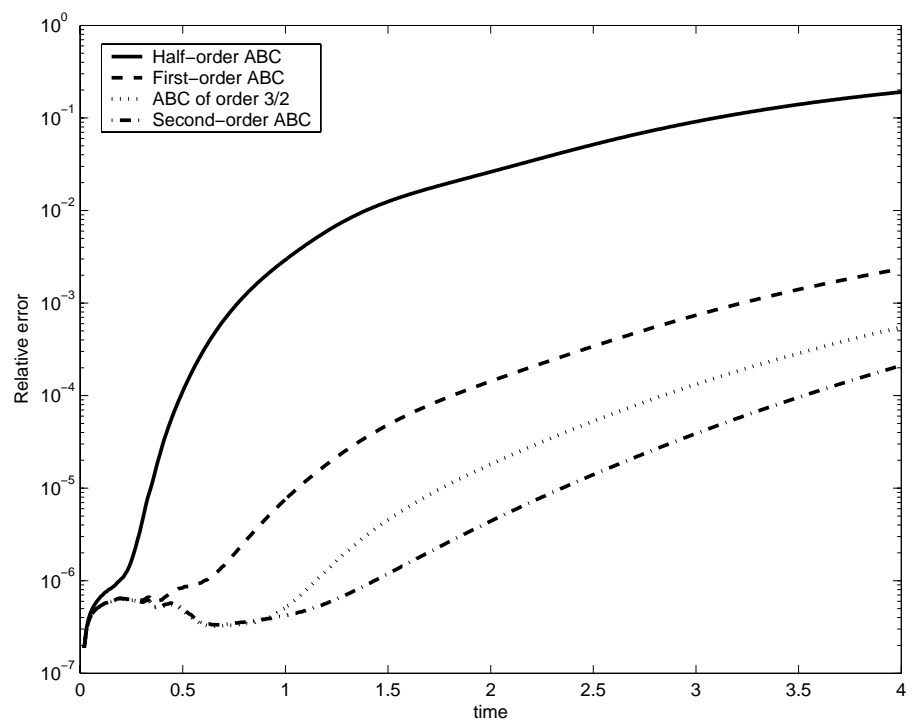

FiguRE 8. Relative error for the gaussian solution $k_{0}=0$. 


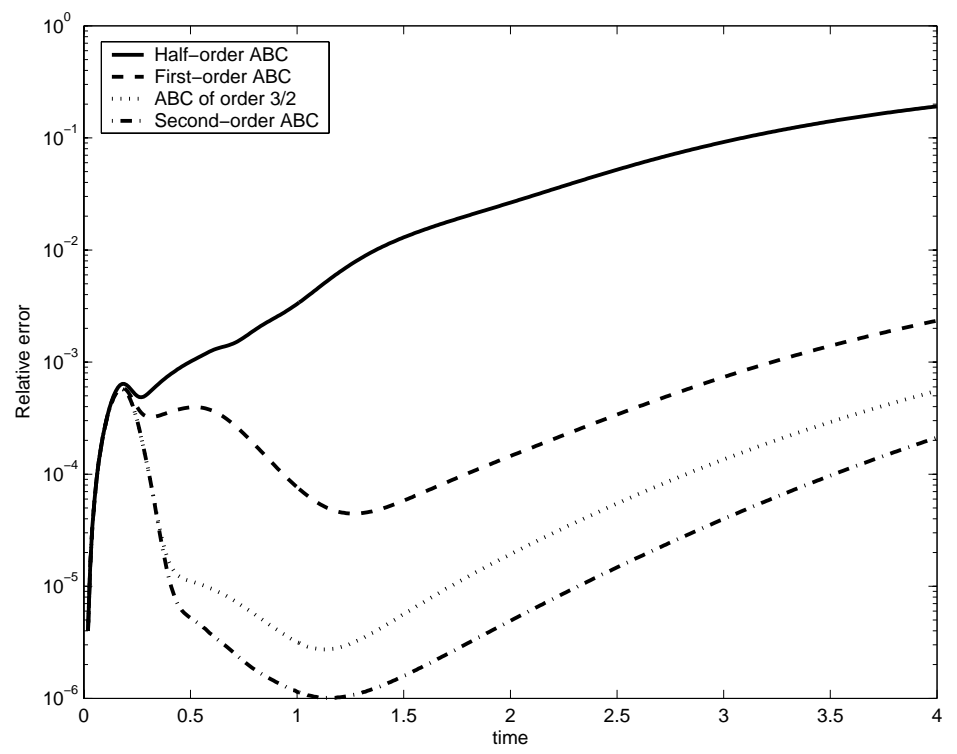

FiguRE 9. Relative error for the superposition of a gaussian solution $\left(k_{0}=0\right)$ and the exact solution for $k_{0}=5$.

Finally, in Figure 10 we perform the evolution of the approximate solution at different times using the second-order artificial boundary condition for the second computational domain. This shows that the method allows us to treat some problems involving a general smooth domain of computation. Only some reflections of small amplitude appear. The behaviour is closed to the one already observed in the previous case.
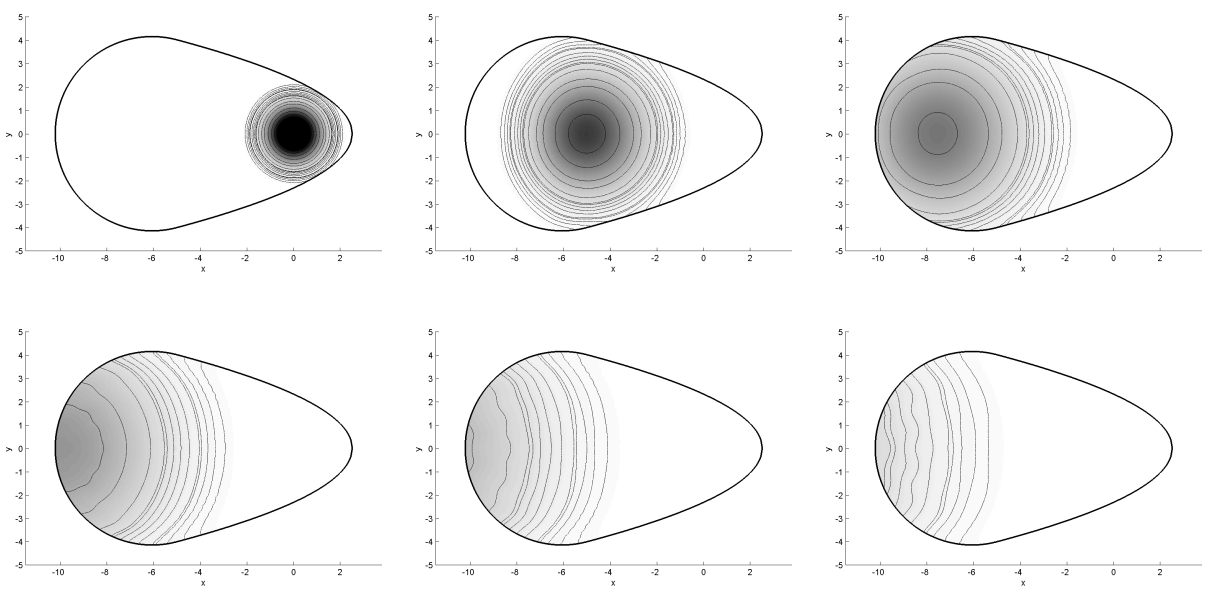

Figure 10. Evolution of the approximate solution at times $t=0$, $t=0.5, t=0.75, t=1, t=1.25$, and $t=1.5$ using the secondorder artificial boundary condition. 


\section{REFERENCES}

[1] I. Alonso-Mallo, and N. Reguera, Weak ill-posedness of spatial discretizations of absorbing boundary conditions for Schrödinger-type equations, SIAM J. Numer. Anal. 40 (1) (2002), pp. 134-158. MR 2003f:65159

[2] X. Antoine, Fast approximate computation of a time-harmonic scattered field using the On-Surface Radiation Condition method, IMA J. Appl. Math. 66 (2001), pp. 83-110. MR 2001m:78033

[3] X. Antoine, and C. Besse, Construction, structure and asymptotic approximations of a microdifferential transparent boundary condition for the linear Schrödinger equation, J. Math. Pures Appl. 80 (7) (2001), pp. 701-738. MR 2003h:35213

[4] X. Antoine, and C. Besse, Unconditionally stable discretization schemes of non-reflecting boundary conditions for the one-dimensional Schrödinger equation, J. Comput. Phys. 181 (1) (2003), pp. 157-175.

[5] A. Arnold, Numerically absorbing boundary conditions for quantum evolution equations, VLSI Design 6 (1-4) (1998), pp. 313-319.

[6] A. Arnold, Mathematical concepts of open quantum boundary conditions, Transport Theory Statist. Phys., 30 (4-6) (2001), pp. 561-584.

[7] A. Arnold, and M. Erhardt, Discrete transparent boundary conditions for wide angle parabolic equations in underwater acoustics, J. Comput. Phys. 145 (1998), pp. 611-638. MR 99g:76084

[8] A. Arnold, and M. Erhardt, Discrete transparent boundary conditions for the Schrödinger equation, Riv. Math. Univ. Parma 6 (4) (2001), pp. 57-108. MR 2003a:35161

[9] V.A. Baskakov and A.V. Popov, Implementation of transparent boundaries for the numerical solution of the Schrödinger equation, Wave Motion 14 (1991), pp. 123-128. MR 92g:78001

[10] C.-H. Bruneau, L. Di Menza, and T. Lerhner, Numerical resolution of some nonlinear Schrödinger-like equations in plasmas, Numer. Methods Partial Differential Equations (1999), pp. 672-696. MR 2000f:82099

[11] P. Deuflhard, and F. Schmidt, Discrete transparent boundary conditions for the numerical solution of Fresnel's equation, Comput. Math. Appl. 29 (9) (1995), pp. 53-76. MR 95k:65083

[12] L. Di Menza, Transparent and absorbing boundary conditions for the Schrödinger equation in a bounded domain, Numer. Funct. Anal. Optim. 18 (7-8) (1997), pp. 759-775. MR 98h:65036

[13] T. Fevens, and H. Jiang, Absorbing boundary conditions for the Schrödinger equation, SIAM J. Sci. Comput. 21 (1) (1999), pp. 255-282. MR 2000h:65144

[14] T. Friese, F. Schmidt, and D. Yevick, A comparison of transparent boundary conditions for the Fresnel equation, J. Comput. Phys. 168 (2001), pp. 433-444. MR 2002b:78027

[15] R. Gorenflo, and F. Mainardi, Fractional Calculus: Integral and Differential Equations of Fractional Order, in Fractals and Fractional Calculus in Continuum Mechanics, Ed. A. Carpinteri and F. Mainardi, Springer-Verlag, Wien 1997. MR 99g:26015

[16] T. Ha Duong, and P. Joly, A generalized image principle for the wave equation with absorbing boundary condition and application to fourth order schemes, Numer. Methods Partial Differential Equations 10 (1994), (4), pp. 411-434. MR 95f:65164

[17] T. Hagstrom, Radiation boundary conditions for the numerical simulation of waves, Acta Numer. (1999), pp. 47-106. MR 2002c:35171

[18] P. Henrici, Fast Fourier methods in computational complex analysis, SIAM Rev. 21 (4) (1979), pp. 481-541. MR 80i:65031

[19] Y.V. Kopylov, A.V. Popov, and A.V. Vinogradov, Application of the parabolic wave equation to X-ray diffraction optics, Optics Comm. 118 (1995), pp. 619-636.

[20] J.-P. Kuska, Absorbing boundary conditions for the Schrödinger equation on finite intervals, Phys. Rev. B 46 (8) (1992), pp.5000-5003.

[21] M. Lévy, Parabolic Equation Methods for Electromagnetic Wave Propagation, IEE, 2000. MR 2003b:78001

[22] C. Lubich, Discretized fractional calculus, SIAM J. Math. Anal. 17 (3) (1986), pp. 704-719. MR 87f:26006

[23] C. Lubich, and A. Schädle, Fast convolution for non-reflecting boundary conditions, SIAM J. Sci. Comput. 24 (1) (2002), pp. 161-182. MR 2003h:44007

[24] B. Mayfield, Non Local Boundary Conditions for the Schrödinger Equation, Ph.D. Thesis, University of Rhodes Island, Providence, RI, 1989. 
[25] A. Schädle, Non-reflecting boundary conditions for the two-dimensional Schrödinger equation, Wave Motion 35 (2002), pp. 181-188. MR 2002h:78010

[26] F. Schmidt, Construction of discrete transparent boundary conditions for Schrödinger-type equations, Surveys Math. Indust. 9 (2) (1999), pp. 87-100. MR 2000j:65080

[27] F. Schmidt, and D. Yevick, Discrete transparent boundary conditions for Schrödinger-type equations, J. Comput. Phys. 134 (1997), pp. 96-107. MR 98e:81028

[28] F.D. Tappert, The parabolic approximation method, in J.B. Keller and J.S. Papadakis, Ed., Wave Propagation and Underwater Acoustics, Lecture Notes in Physics, Vol. 70, pp. 224-287, Springer, Berlin, 1977. MR 57:14891

[29] A.I. Zayed, Handbook of Function and Generalized Function Transformation, CRC Press, 1996. MR 97h:44001

Laboratoire de Mathématiques pour l'Industrie et la Physique, CNRS UMR 5640, UFR MiG, 118, route de Narbonne, 31062 Toulouse Cedex 4, France

E-mail address: antoine@mip.ups-tlse.fr

Laboratoire de Mathématiques pour l'Industrie et la Physique, CNRS UMR 5640, UfR Mig, 118, route de Narbonne, 31062 Toulouse Cedex 4, France

E-mail address: besse@mip.ups-tlse.fr

Laboratoire de Mathématiques pour l'Industrie et la Physique, CNRS UMR 5640, UfR Mig, 118, route de Narbonne, 31062 Toulouse Cedex 4, France

E-mail address: mouysset@mip.ups-tlse.fr 